\title{
Fast Adaptive Upscaling of Low Designed Images through a Categorized Filling Strategy
}

\author{
Qurratul Aini ${ }^{1} \mid$ Sabah Syed Nasirullah ${ }^{2}$ | Sumaya Tazeen ${ }^{3}$ \\ ${ }^{1}$ Dept ECE, Research Scholar, Shri JJTU university, Rajasthan, India \\ ${ }^{2}$ Dept ECE, Research Scholar, Shri JJTU university, Rajasthan, India \\ ${ }^{3}$ Dept ECE, Assistant Professor, SWCET, Hyderabad, Telangana, India
}

\section{To Cite this Article}

Qurratul Aini, Sabah Syed Nasirullah and Sumaya Tazeen, "Fast Adaptive Upscaling of Low Designed Images through a Categorized Filling Strategy", International Journal for Modern Trends in Science and Technology, 6(8S): 17-19, 2020.

\section{Article Info}

Received on 16-July-2020, Revised on 15-August-2020, Accepted on 25-August-2020, Published on 28-August-2020.

\section{ABSTRACT}

Image upscaling approaches is required particularly when calculations are applied to subsampled pictures so as to accelerate handling time. "Customary strategies for upscaling pictures yield frequently obscured or blocky outcomes yet more confounded strategies are tedious". "We present a versatile upscaling approach for low organized pictures, which gives continuous capacity to pictures in full TV goal and creates clear and non-blocky edges because of a progressive filling methodology".

Key words: adaptive interpolation, subsampling, real time video processing, binary mask interpolation, dense disparity estimation

\section{INTRODUCTION}

Different applications need interjection approaches for resizing pictures. "While resizing pictures the principle challenge is the manner by which to reproduce satisfactory qualities for missing pixels in the enormous picture utilizing data from pixels in the little picture". "Regularly utilized methodologies here are closest neighbor technique, bilinear interjection and bicubic insertion [11-7]". "One fundamental disadvantage of every one of these strategies, in any case, is that they produce either blocky edges (closest neighbor) or obscured pictures (bilinear and bicubic interjection)". "Moreover, if there is an extra continuous limitation, these procedures are excessively perplexing just as being excessively moderate". "Accordingly we present a novel methodology, particularly for low organized pictures or parallel pictures, which depends on a quick addition strategy utilizing another various leveled filling procedure for safeguarding clear edges while giving continuous capacity in full TV goal video groupings".

\section{HIERARCHICAL FILLING STRATEGY}

"The principle issue with ordinary addition strategies is that they apply the equivalent introduction system to the entire picture". "Thus, expensive methods can't be acknowledged in continuous while basic strategies don't arrive at adequate quality". "In this way we propose an versatile methodology, which recognizes picture areas of various criticality". "In our approach a computationally escalated and edge saving procedure is just acted in basic picture territories, though basic and all the more straight-forward techniques are utilized in the remaining districts". "The versatile insertion approach we are introducing in this paper works 
blockwise". "For a NxN up-testing factor $\mathrm{NxN}$ squares are inserted by handling $(\mathrm{N}+1) \times(\mathrm{N}+1)$ covering windows". "In each square the pixel esteems at the corners are known from the little picture (see Fig. 1 and Fig. 3)". "For deciding the missing pixel esteems in current square just pixels inside this square are thought of the choice on an appropriate introduction method is made by dissecting the relationship of the corner pixels in a square". "On the off chance that each of the four values are comparable, or even equivalent, a standard insertion strategy method is utilized". "In any case a various levelled filling strategy is applied". "For this last strategy it is expected that the picture is low organized, for example there are at the most extreme two districts in a square, speaking to the different sides of an edge". "Moreover it must be considered, that the square size, speaking to the up-testing factor is an intensity of two". "In the following sections two models are introduced,portraying the various levelled interjection approach for a double picture case and a dark worth picture case, individually".

\subsection{Filling Binary Images}

In Fig. 1 the up-sampling of a binary image with an up-sampling factor 4 is represented.

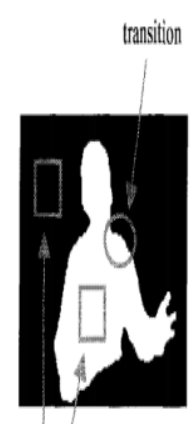

homogenous region

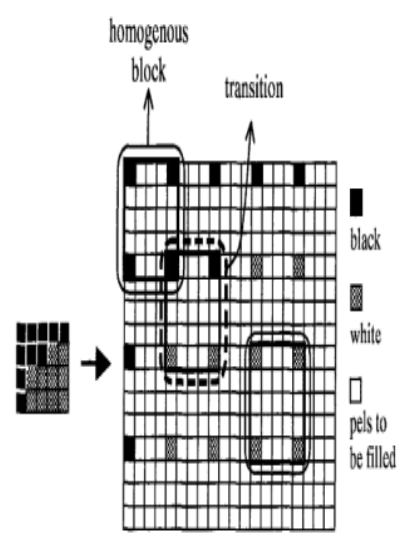

Fig. 1: Interpolation of a binary image

"Homogeneous and in homogenous territories are stamped calling attention to two diverse filling strategies". "Homogeneous areas perceived by identifying indistinguishable corner pixels in a square, are filled by a straightforward duplicate of the reference pixel esteem into the missing middle pixels". "In in homogenous locales in any event one of the 4 corner pixels varies from the others (Fig. 2)". "In this case filling functions as follows the primary line and the principal section in a window with differing corner pixels are known from recently handled covering squares".

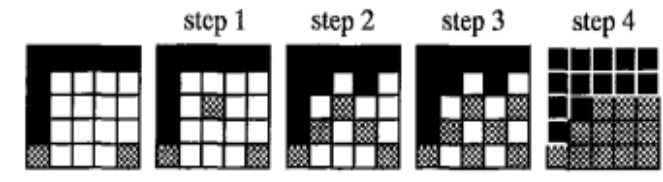

Fig. 2: Hierarchical filling of a binary block

"As portrayed in Fig. 2, in the initial step of the various levelled filling the pixel at focal square position is filled (stamped dim in sync 1 of Fig. 2)". "Here just the 4 corner pixels are taken as choice possibility for a dominant part choice rule". "In the event that the outcome is uncertain, two arrangements are conceivable the first is to choose one mark ahead of time before the entire handling and to rule for this name in such circumstances". "The other is to take extra data into account, if conceivable, obviously relying upon the application". "As a result of the way that this center pixel conveys a very critical impact on the succeeding handling, it is prescribed to contemplate additional data (for example outline contrast at this mid-point position on account of progress identification or division issues)". "In the second step a similar system is rehashed for the four coming about sub-squares, bringing about thefour new mid-position pixels". "For every one of these pixels the choice is made on premise of the two direct neighbors in corner to corner heading". "In the third step just level and vertical neighbors are thought of in the last advance for every pixel each of the four direct neighbors (two in level and two vertical way) are thought of note that, subsequently emphatically various leveled system, the addition is quick and conveys a definitive and smooth edge".

\subsection{Filling Gray Value Images}

Fig. 3 shows the up-sampling of a gray value image with an up-sampling factor of 4 .

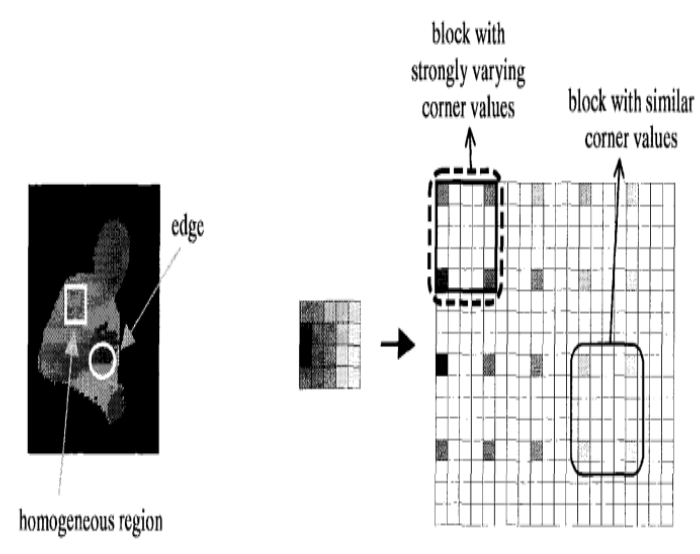

Fig. 3: Interpolation of a gray value image 
"In Fig. 3 (left) a dissimilarity map is appeared for instance for a low-organized picture". "On the right one window with very unique corner pixels and one window with comparable corner pixels are picked". "An edge for the contrast between the most minimal and most noteworthy corner pixel must be characterized so as to conclude whether to regard the square as a homogeneous or an in homogenous square". "Homogeneous squares can be filled for example by bilinear interjection or closest neighbor approach expecting a uniform zone". "In homogenous squares are filled progressively as appeared in Fig. 4".

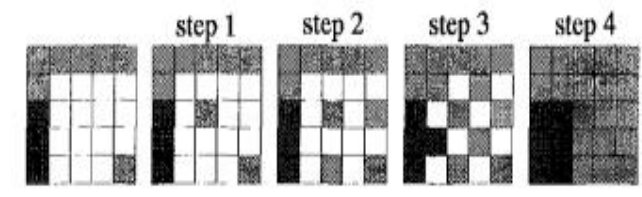

Fig. 4: Hierarchical filling of a gray value block

\subsection{Experimental Results}

"Fig. 5 shows test results from two unique applications". "The parallel pictures show covers because of a division procedure on CCIRBOI position, which has been applied to the subsampled picture to accelerate the preparing". "The center picture shows the resizing result utilizing closest neighbor, though on the privilege the non-blocky consequence of various leveled filling is introduced".

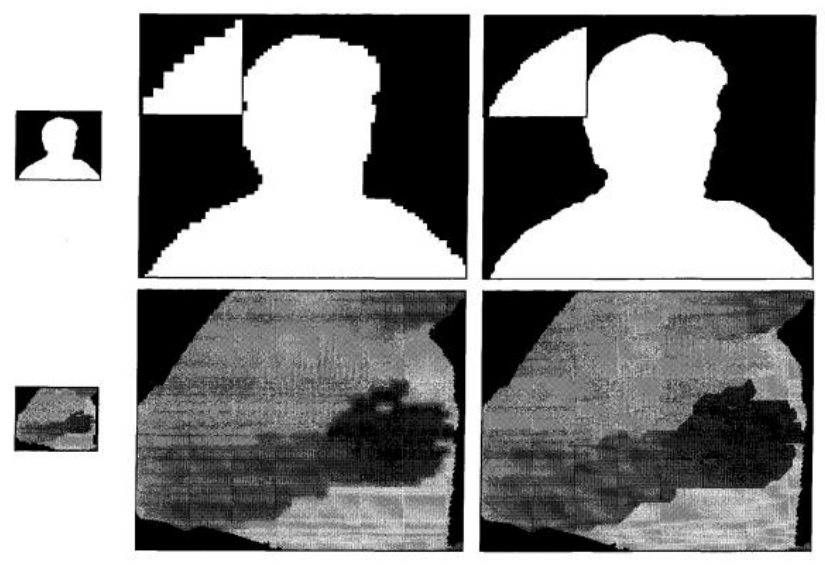

Fig. 5: Top: segment masks, Bottom: depth maps; subsampled images (left), simple interpolation (middle), hierarchical approach (right)

"The dark worth pictures show up-examining aftereffects of a uniqueness map (left), which has been assessed on a $8 \times 8$ matrix". "While the center picture made by bilinear interjection shows obscuring impacts, the correct outcome jam edges clear and adds homogeneous territories"."In [8] for instance this various leveled addition".

\section{CONCLUSION}

"We introduced a novel methodology for quick up scaling of low organized pictures, which produces smooth however clarified and non-blocky edges". "This objective is accomplished by the versatile character of the methodology applying a computationally concentrated technique just in basic regions and a basic technique in different regions". "In the basic districts taking care of in a various levelled request, and for dark worth pictures utilizing a middle sifting base, jelly edges clear". "In different districts a less expensive technique essentially introduces the region".

\section{REFERENCES}

[1] http://www.fmde.com/multi-tech-861O-image-interpolatio n_methods.htm

[2] J.-R. Ohm, "Digitale Bildcodierung", Springer-Verlag, Berlin, 1995

[3] J.-R. Ohm, "Bildverarbeitung 11", Skript, TU-Berlin, Institut fiir Nachrichtentechnik und Theoretische Elektrotechnik, 1999

[4] http://www.pcigeomatics.com/cgi-bin/pcihlp/REGlBACK GROUND

[5] http://www.cee.hw.ac.uk/hipr/html/scale.html

[6] http://www.dpreview.com/learn/Glossary/Digital_Imagin $\mathrm{g} /$ lnterpolation-01.htm

[7] http://www.mathworks.com/access/helpdesk/help/toolb ox/images/geom2.shtml

[8] O. Schreer, N. Brandenburg, S. Askar, P. Kauff, "Hybrid Recursive Matching and Segmentation-Based Postprocessing in Real-Time hmersive Video Conferencing", Proc. of VMV 2001, Vision, Modeling and Visualization 2001, Stuttgart, Germany, Nov. 2001 